\title{
Omeprazole in the treatment of asthmatics with nocturnal symptoms and gastro-oesophageal reflux: a placebo-controlled cross-over study
}

\author{
G.A. Ford, P.S. Oliver, J.S. Prior, R.J.A. Butland and S.P. Wilkinson
}

Department of Medicine, Gloucestershire Royal Hospital, Great Western Road, Gloucester GL1 3NN, UK

\begin{abstract}
Summary: Eleven adult patients with nocturnal asthma, and gastro-oesophageal reflux documented by endoscopy or ambulatory oesophageal pH monitoring completed a double-blind cross-over study (4 week treatment, one week run-in and cross-over periods) comparing the effects of omeprazole $20 \mathrm{mg}$ daily and placebo on asthma control assessed by symptoms, peak expiratory flow rate and bronchodilator usage. Omeprazole treatment did not improve asthma symptoms during the day or night, or peak expiratory flow rate readings. There was no difference in bronchodilator inhaler usage during omeprazole therapy.

Treatment of gastro-oesophageal reflux with omeprazole in patients with nocturnal asthma and gastro-oesphageal reflux does not improve asthma symptoms or peak expiratory flow rate. This suggests that gastro-oesophageal reflux does not exacerbate bronchoconstriction in nocturnal asthma.
\end{abstract}

\section{Introduction}

It has been suggested that gastro-oesophageal reflux (GOR) may be an important cause or exacerbating factor in nocturnal asthma, although this is subject to considerable controversy. ${ }^{1-10}$ Recent studies indicate that $39 \%$ of asthmatics have oesophagitis or Barrett's oesophagus ${ }^{11}$ and $80 \%$ of asthmatics have abnormal GOR. ${ }^{12}$ Although bronchodilator therapy could lead to GOR, these studies suggest that this high incidence cannot be accounted for by bronchodilator therapy alone. GOR could lead to bronchoconstriction by either micro aspiration directly producing bronchoconstriction, or stimulation of afferent vagal nerves increasing bronchoconstriction or airway responsiveness to extrinsic allergens. ${ }^{3,13}$ In support of the latter theory, there is evidence that vagal mechanisms are fundamentally involved in the pathophysiology of nocturnal asthma.$^{14}$ This might be particularly noticeable at night as pathological GOR is often more severe in the supine position.

Anecdotal case reports have suggested that $\mathrm{H} 2$ receptor antagonists or surgery may be beneficial in reducing asthmatic symptoms. ${ }^{15}$ One randomized unblinded study found some minor improvement in asthma symptoms in patients with oesophageal symptoms who received advice on conservative management of GOR without the use of $\mathrm{H} 2$ receptor antagonists. ${ }^{16}$ There are three placebo-

Correspondence: S.P. Wilkinson, F.R.C.P., Derriford Hospital, Derriford Road, Plymouth PL6 8DH, UK. Accepted: 29 October 1993 controlled trials of the effect of $\mathrm{H} 2$ receptor antagonists in asthmatic patients with GOR. One demonstrated a small increase in evening peak expiratory flow rate and a small improvement in nocturnal respiratory symptoms. ${ }^{17}$ Ekstrom et al. also demonstrated a small improvement in nocturnal asthma symptoms but found no change in peak expiratory flow rate, bronchial reactivity or lung function. ${ }^{18} \mathrm{~A}$ third study found no benefit in respiratory symptoms or peak flow. ${ }^{19}$ One case report has described improvement of nocturnal asthma with omeprazole, a much more effective treatment for GOR, ${ }^{20}$ in a patient with GOR who had shown no improvement with high-dose ranitidine. ${ }^{21}$ Because these previous studies utilizing less effective therapies for GOR produced equivocal results, we undertook a placebo-controlled study of omeprazole in patients with nocturnal asthma and GOR.

\section{Methods}

\section{Study design}

The study was a double-blind, randomized, crossover trial comparing omeprazole $20 \mathrm{mg}$ once daily with placebo in patients with nocturnal asthma and gastro-oesophageal reflux. The 10-week study period consisted of a one-week run-in followed by two 4-week treatment periods, separated by a one-week wash-out. The primary therapeutic end point studied was peak expiratory flow rate with 
asthma symptoms and bronchodilator usage as secondary end-points. Patients were seen in the clinic at the end of the placebo run-in period (end of week one) and at the end of each treatment period (weeks 5 and 10). At each visit symptoms of day-time asthma (no problems, wheezy for $<2$ hours a day, wheezy most of day, wheezy all day) and night-time (undisturbed, slept well but woke feeling wheezy, woken 2-3 times a night because of asthma, awake most of night with asthma) were assessed on a scale of $0-3$. Patients were issued with diary cards to record day-time and night-time asthma symptoms as above, peak expiratory flow rate (PEFR) on waking before using bronchodilator, PEFR between 4 and $6 \mathrm{pm}$, on PEFR 30 minutes before retiring to bed, whether trial medication was taken and use of inhaled B2adrenoceptor agonist drugs. Symptoms were recorded before PEFR was measured. Symptoms of GOR were also recorded at each visit at the end of the run-in, omeprazole and placebo periods. Symptoms were assessed on a scale $0-3$ as follows: heartburn (none, occasional episodes not interfering with normal activities, occasional interference with normal activities, and constant interference); and regurgitation (none, occasional on straining or lying down, predictable on position or straining, and pulmonary aspiration manifested by recurrent pneumonia).

\section{Subjects}

Eleven patients with nocturnal asthma and evidence of gastro-oesophageal reflux were recruited to the study from respiratory outpatient clinics. Patients (five female, two smokers) had a mean age of 63 (range 50-80) years. Mean weight was 74 $(53-109) \mathrm{kg}$ and height $168(152-180) \mathrm{cm}$. The duration of asthma symptoms was $11(0.8-48)$ years. Duration of nocturnal asthma symptoms was $3.4(0.7-14)$ years. The mean duration of GOR symptoms was $52(7-99)$ months and the duration of verified reflux oesophagitis was 6.4 $(0.1-50)$ months. One patient withdrew because of uncontrolled asthma symptoms requiring treatment with oral prednisolone (between visits two and three) and was excluded from the analysis. Asthma medication, except for as required inhaled $\beta 2$-adrenoceptor agonists, was constant for 2 weeks prior to and during the study. Eight patients were taking inhaled steroids and one patient inhaled ipatroprium. Use of antacids but not $\mathrm{H} 2$ antagonists, metoclopramide or other anti-reflux drugs was allowed during the study period. Other concomitant medication taken throughout the study was bismuth (two patients), frusemide (1), bendrofluazide (1), amitriptylline (1), metronidazole (1), tetracycline (1) and lorazepam (1).

All patients had a history of asthma symptoms for at least 6 months and met one of the three following criteria for nocturnal asthma: asthmatic symptoms (cough, wheeze) causing nocturnal waking at least three nights a week, awakening with morning chest tightness on at least three mornings a week, or wakening with at least a $10 \%$ dip in PEFR compared to previous night at least three times a week. All patients also had at least a $15 \%$ increase in PEFR 15 minutes following inhaled terbutaline or salbutamol. PEFR before and after inhaled terbutaline was $253 \pm 83$ (range 100-360) $1 /$ minute and $308 \pm 94(130-414) 1 /$ minute, a $23 \pm 8 \%$ increase in PEFR.

All patients met the following criteria for GOR: endoscopically proven oesophagitis and/or abnormal GOR on 24 hour ambulatory oesophageal pH monitoring as defined by DeMeester et al. ${ }^{22}$ Upper gastrointestinal endoscopy was performed in all 11 patients and 24 hour ambulatory oesophageal pH monitoring was performed in nine prior to study entry. Two patients with grade 3 oesophagitis at endoscopy did not have 24 hour ambulatory oesophageal $\mathrm{pH}$ monitoring undertaken. Six patients had macroscopic oesophagitis at endoscopy according to the Savery-Miller classification: one Grade 1, one Grade II, and four Grade III. Two patients had a Barrett's oesophagus, defined as columnar lined epithelium extending more than $3 \mathrm{~cm}$ above gastro-oesophageal junction. No patient had a stricture or had previously undergone oesophageal dilatation. No patients were taking theophylline derivatives or oral $\beta 2$-adrenoceptor agonists. Full blood count, routine biochemistry and urinalysis were performed prior to study entry; no significant abnormalities were found. Three patients received antibiotic therapy during the study period.

\section{Data analysis}

An intention to treat analysis was undertaken, although data from the patient who withdrew because of oral steroid therapy were excluded because of insufficient data in both periods. The methods described by Hills and Armitage were used..$^{24,25}$

\section{Results}

Compliance measured by return tablet counts in the 10 patients who completed the study was $96 \%$. Initial analysis of the data revealed no significant treatment, period or carry-over effects. The results of patient recorded morning PEFR and bronchodilator usage recorded on diary cards daily are shown in Figure 1. There were no significant differences between placebo and omeprazole therapy for any of the variables assessing asthma 
symptoms, bronchodilator usage and PEFR. Early morning reduction peak expiratory flow rate remained at around $301 /$ minute throughout the study. PEFR was unchanged by omeprazole on waking $(262 \pm 86$ versus $255 \pm 861 /$ minute, mean \pm s.d., omeprazole versus placebo), during the daytime $(293 \pm 80$ versus $291 \pm 81)$ and at night $(280 \pm 81$ versus $277 \pm 78)$. Night-time $(1.0 \pm 0.6$ versus $1.0 \pm 0.7)$ and day-time $(1.0 \pm 0.7$ versus $0.9 \pm 0.7$ ) asthma symptom scores were similarly not altered by omeprazole. Night-time and day-time bronchodilator usage was not significantly reduced by omeprazole (night-time $0.8 \pm 1.2$ versus $1.0 \pm 1.7$; day-time $4.7 \pm 2.8$ versus $6.0 \pm 4$ ) (Figure 2). The statistical power of the test is $29 \%$ and the small sample size may have been unable to detect a beneficial treatment effect on day-time bronchodilator use. Results from 20 patients in each group would be required to detect a true difference of 1.2 puffs/day at $80 \%$ power and a $5 \%$ significance level. Seven of 11 patients recorded a treatment preference for omeprazole and none for placebo $(P<0.01)$. There was a trend for reflux symptoms to improve during omeprazole therapy. Number of patients with no symptoms in each treatment group was: heartburn (run-in 1, placebo 3 , omeprazole 5), regurgitation (run-in 3, placebo 3 , omeprazole 3 ). The changes in GOR symptom scores were not significantly different.

\section{Discussion}

The results of this study show no significant improvement in control of nocturnal asthma as assessed by symptoms, PEFR and bronchodilator usage following treatment with omeprazole. Because of the small number of patients studied, a beneficial effect of omeprazole with a small improvement in asthma control with decreased daytime inhaler usage, might not have been detected in this study. We chose to study a highly selected group of asthmatic patients, those with nocturnal symptoms and documented GOR, in the belief that this group of asthmatic patients were the most likely to have GOR as a significant aetiological factor in producing nocturnal dipping. Our observations argue against GOR being a significant aetiological factor in morning dipping. No significant difference in symptoms of GOR was found in this study, although this was not a primary endpoint of interest in the study and is almost certainly due to the use of a non-continuous variable in the number of patients studied, unlike the measures of asthma control which were analysed as continuous variables (PEFR readings or mean diary scores over 4 or 7 days). The doubling of patients with no symptoms of heartburn or regurgitation during omeprazole therapy is

\section{MORNING PEAK EXPIRATORY FLOW RATE}

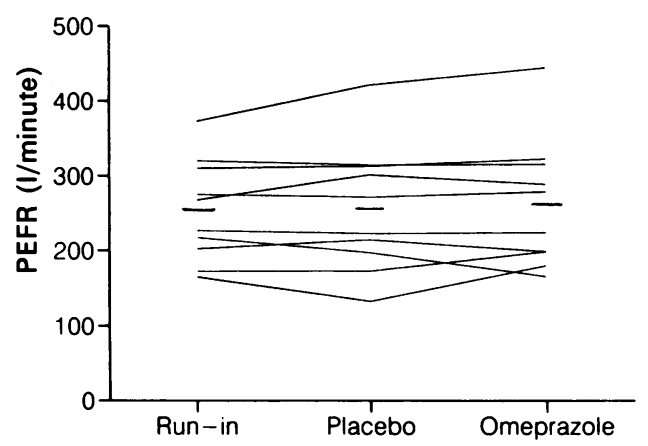

Figure 1 Morning peak expiratory flow rate recorded on waking in diary cards during run-in, placebo and omeprazole treatment periods. Data are individual values and mean (horizontal bars) in 10 patients collected over either 4 days (run-in) or 7 days (placebo/omeprazole).

consistent with the results of omeprazole's efficacy in the treatment of GOR in larger studies. The use of diary cards or visual analogue scales to assess GOR symptoms or repeated 24 hour ambulatory oesophageal $\mathrm{pH}$ measurements would have provided more accurate assessment of the effect of omeprazole on GOR in these patients.

It remains possible that a larger dose of omeprazole might have produced a beneficial effect. Omeprazole at a dose of $\mathbf{4 0} \mathrm{mg}$ daily results in more prolonged acid suppression. ${ }^{15}$ However, clinical trials comparing the effect of $20 \mathrm{mg}$ and $40 \mathrm{mg}$ omeprazole show only a slight therapeutic advantage in healing oesophagitis and it is unlikely that using $40 \mathrm{mg}$ omeprazole to treat GOR in asthmatics would bring about a significant difference in asthma. ${ }^{26,27}$ Another possibility is that a longer treatment period is required to reverse any effect of GOR on vagally mediated bronchial hyperresponsiveness. In support of this the one small study to show a reduction in asthma symptoms used 6 weeks ranitidine therapy. ${ }^{17}$ Studies undertaking repeated measurements of bronchial hyperresponsiveness during prolonged omeprazole therapy would address this tissue. A further possibility is that omeprazole failed to show any benefit on asthma control because a component of refluxed gastric fluid other than acid is responsible for increasing bronchial hyper-responsiveness. Again, this is an unlikely possibility as many studies have demonstrated that acid instilled into the oesphagus and not saline or alkali, result in bronchoconstriction. ${ }^{28}$ Finally, it remains possible that treatment of GOR is ineffective because the GOR in asthmatics is a secondary phenomenon related to changes in intrathoracic pressure. The 

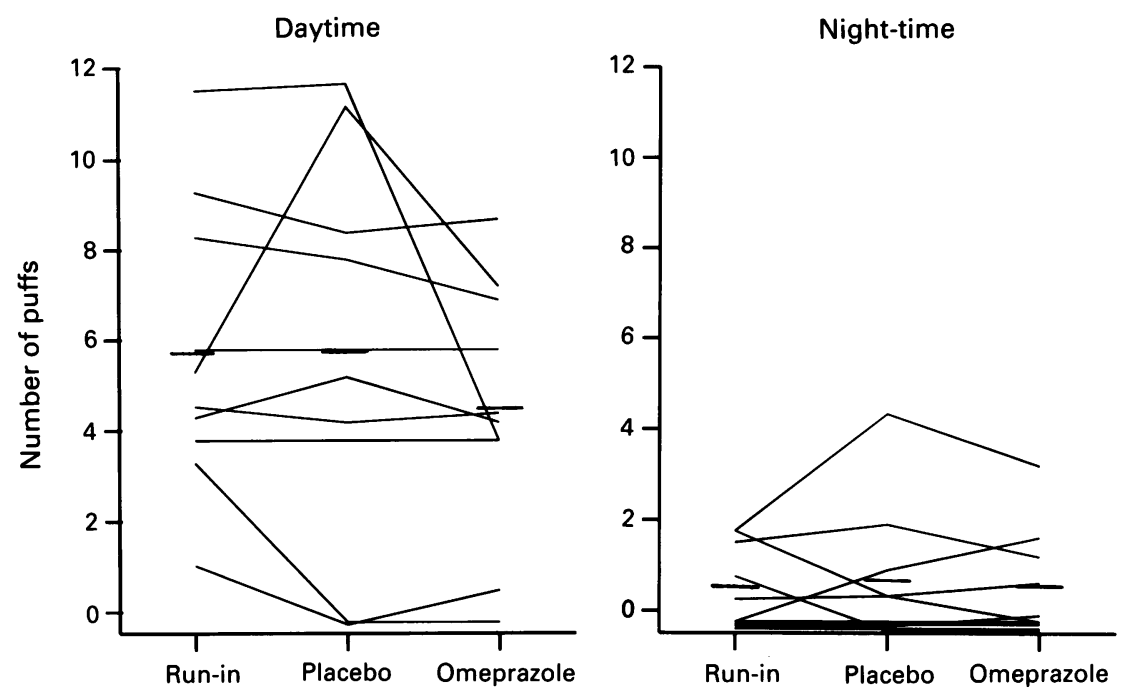

Figure 2 Daytime and night-time bronchodilator usage recorded in diary cards during run-in, placebo and omeprazole periods. Data are individual values and mean in 10 patients collected over either 4 days (run-in) or 7 days (placebo/omeprazole).

shorter history of GOR compared to asthma symptoms in our patients would lend support to this concept. Both asthma and GOR are common in the population (approximately $15 \%$ and $8 \%$ incidence, respectively) and they would be expected to coexist in some individuals, although the high prevalence of GOR documented in studies of unselected asthmatics indicates this cannot be the sole explanation..$^{11,12}$ Studies of the prevalence of GOR in patients with asthma sugguest more than $80 \%$ asthmatics have abnormal GOR. Bronchodilator use may exacerbate GOR, although one study found that bronchodilator therapy did not influence the prevalence of GOR. ${ }^{11,12}$

Our results in conjunction with those of previous studies suggest that GOR is at most only a minor factor in exacerbating asthma symptoms. Ekstrom et al. found that a history of reflux associated symptoms appeared to be the only factor that identified which patients' asthma would benefit from treatment for GOR. ${ }^{14}$ Case reports suggest that in individual patients GOR may be a major causative factor in producing bronchoconstriction, although in many of these cases GOR was severe and gross aspiration may have occurred. Despite the association between asthma and GOR, our results in conjunction with previous studies suggest that routine investigation for GOR in asthmatic patients is not indicated, as treatment of GOR is unlikely to result in significant improvement in asthma control.

\section{Acknowledgement}

We thank Astra Pharmaceuticals for providing the drugs for this study and assistance with statistical analysis.

\section{References}

1. Anonymous. Gastric asthma. Lancet 1985 , ii: $1399-1400$.

2. Goldman, J.M. \& Bennett, J.R. Gastro-oesophageal reflux and asthma; a common association, but of what clinical importance? Gut 1990, 31: 1-3.

3. Tuchman, D.N., Boyle, J.T. \& Pack, A.I. Comparison of airway responses following tracheal or oesophageal acidification in the cat. Gastroenterology 1984, 87: 872-881.

4. May, E.E. Intrinsic asthma in adults. Association with gastro-oesophageal reflux. JAMA 1976, 236: 2626-2628.
5. Harper, P.C., Bergner, A. \& Kaye, M.D. Anti-reflex treatment for asthma. Improvement in patients with associated gastro-oesphageal reflux. Arch Intern Med 1987, 147: 56-60.

6. MacDonald, J.B. Nocturnal asthma. Br Med J 1992, 304 998-999.

7. Ekstrom, T. \& Tibbling, L. Gastro-oesophageal reflux and nocturnal asthma. Eur Respir J 1988, 1: 636-638. 
8. Ekstrom, T. \& Tibbling, L. Gastro-oesophageal reflux and triggering of bronchial asthma: a negative report. Eur $J$ Respir Dis 1987, 71: 177-180.

9. Pack, A.L. Acid: a nocturnal bronchoconstrictor? Am Rev Respir Dis 1990, 142: 1153-1157.

10. Mansfield, I.E. Gastro-oesphageal reflux and respiratory disorders: a review. Ann Allergy 1989, 62: 158-163.

11. Sontag, S.J., Miller, T.Q., Khandelwal, S. et al. Prevalence of oesophagitis in asthmatics. Gut 1992, 33: 872-876.

12. Sontag, S.J., O'Connell, S., Khandelwal, S. et al. Most asthmatics have gastroesophageal reflux with or without bronchodilator therapy. Gastroenterology 1990, 99: 613-620.

13. Mansfield, L.E. \& Stein, M.R. Gastro-oesphageal reflux and asthma, a possible reflex mechanism. Ann Allergy 1978, 41: 224-226.

14. Morrison, J.F.J., Pearson, S.B. \& Dean, H.G. Parasympathetic nervous system in nocturnal asthma. Br Med J 1988, 296: 1427-1429.

15. Pellegrini, C.A., DeMeester, T.R., Johnson, L.F. \& Skinner, D.B. Gastro-oesophageal reflux and pulmonary aspiration: incidence, functional abnormality and results of surgery. Surgery 1979, 86: 110-118.

16. Kjellen, G., Tibbling, L. \& Wranne, B. Effect of conservative treatment of oesophageal dysfunction on bronchial asthma. Eur J Respir Dis 1981, 62: 190-197.

17. Goodall, R.J.R., Earis, J.E., Cooper, D.N., Bernstein, A. \& Temple, J.G. Relationship between asthma and gastrooesphageal reflux. Thorax 1981, 36: 116-121.

18. Ekstrom, T., Lindgren, B.R. \& Tibbling, L. Effects of ranitidine treatment on patients with asthma and a history of gastro-oesphageal reflux: a double blind crossover study. Thorax 1989, 44: 19-23.

19. Nagel, R.A., Brown, P., Perks, W.H., Wilson, R.S.E. \& Kerr, G.D. Ambulatory $\mathrm{pH}$ monitoring of gastro-oesophageal reflux in 'morning dipper' asthmatics. Br Med J 1988, 296: 1371-1373.
20. Mctavish, D., Buckley, M.M. \& Heel, R.C. Omeprazole. An updated review of its pharmacology and therapeutic use in acid-related disorders. Drugs 1991, 42: 138-170.

21. Depla, A.C., Bartelsman, J.F., Roos, C.M., Tytgat, G.N. \& Jansen, H.M. Beneficial effect of omeprazole in a patient with severe bronchial asthma and gastro-oesophageal reflux. Eur Respir J 1988, 1: 966-968.

22. DeMeester, T.R., Wang, C.I., Wernly, J.A. et al. Techniques, indications and clinical use of 24 hour oesophageal $\mathrm{pH}$ monitoring. J. Thoracic Cardiovasc Surg 1980, 79: 656-670.

23. Savary, M. \& Monnier, P. Rullo dell'endoscopias neela diagnosi delle lesion esofagie de reflusso. In: Barbara, L. \& Bladi, F. (eds) La Malathia da Reflusso Gastroesofageo. Edizione Libreria Cortina, Verona, 1982, pp. 137-166.

24. Hills, M. \& Armitage, P. Two-period cross-over clinical trial. Br J Clin Pharmacol 1979, 8: 7-20.

25. Kenward, M.G. \& Jones, B. The analysis of data from $2 \times 2$ cross-over trials with baseline measurements. Statist Med 1987, 6: 911-926.

26. Robinson, M., Maton, P.N., Allen, M.L. et al. Effect of different doses of omeprazole on 24-hour oesophageal acid exposure in patients with gastro-oesophageal reflux. Aliment Pharmacol Ther 1991, 5: 645-651.

27. Klinkenberg-Knol, E.C., Festen, H.P. \& Meuwissen, S.G. The effects of omeprazole and ranitidine on 24-hour pH in the distal oesophagus of patients with oesophagitis. Aliment Pharmacol Ther 1988, 2: 221-227.

28. Anderson, L., Schmidt, A. \& Bundgaard, A. Pulmonary function and acid application in the esophagus. Chest 1986, 90: $358-363$ 